\title{
The past, present, and future of monoclonal antibodies to IL-5 and eosinophilic asthma: a review
}

\author{
Megan F Patterson' \\ Larry Borish ${ }^{2,3}$ \\ Joshua L Kennedy ${ }^{1,4}$ \\ 'Department of Pediatrics, Arkansas \\ Children's Research Institute, \\ University of Arkansas for Medical \\ Sciences, Little Rock, AR, USA; \\ ${ }^{2}$ Department of Medicine, Carter \\ Immunology Center, University \\ of Virginia Health Systems, \\ Charlottesville,VA, USA; ${ }^{3}$ Department \\ of Microbiology, Carter Immunology \\ Center, University of Virginia Health \\ Systems, Charlottesville, VA, USA; \\ ${ }^{4}$ Department of Internal Medicine, \\ Arkansas Children's Research \\ Institute, University of Arkansas for \\ Medical Sciences, Little Rock, AR, USA
}

Correspondence: Joshua L Kennedy Department of Pediatrics, University of Arkansas for Medical Sciences, I3 Children's Way, Slot 5I2-13, Little Rock, AR 72202, USA

$\mathrm{Tel}+\mathrm{I} 50 \mathrm{I} 3641060$

Fax +I 50I3643I73

Email kennedyjoshual@uams.edu
This article was published in the following Dove Press journal: Journal of Asthma and Allergy

3 November 2015

Number of times this article has been viewed
Abstract: Asthma is a heterogeneous syndrome that might be better described as a constellation of phenotypes or endotypes, each with distinct cellular and molecular mechanisms, rather than as a singular disease. One of these phenotypes is eosinophilic asthma. As the development of eosinophilic inflammation is categorically dependent on the biological activity of Interleukin (IL)-5, IL-5 antagonism became an obvious target for therapy in this phenotype. Early trials of monoclonal antibodies targeting the biological activity of IL-5, including reslizumab, mepolizumab, and benralizumab, were performed on asthmatics with no concern for evidence of eosinophilia. These trials were largely unsuccessful. However, during these trials, researchers recognized the need to quantify eosinophilia in asthma subjects in order to identify those asthmatics in whom these medications would be more likely to improve symptoms and lung function. Using biomarkers, such as sputum and blood eosinophilia, recent studies of these medications have shown improvements in blood and sputum eosinophilia, forced expiratory volume in 1 second, and quality of life assessments as well as reducing occurrences of exacerbations. Moving forward, better and less invasive biomarkers of eosinophilia are necessary to ensure that the correct patients are chosen to receive these medications to receive maximal benefit.

Keywords: eosinophilic asthma, reslizumab, mepolizumab, benralizumab, IL-5, eosinophils

\section{Introduction}

Asthma is a chronic disease with a prevalence of up to $\sim 12 \%$ of the United States population, characterized by reversible airflow obstruction, inflammation, and airway hyperresponsiveness. ${ }^{1-3}$ This disease has many presentations, ranging from mild, intermittent disease to severe, debilitating, even life-threatening symptoms requiring multiple medications, hospitalizations, and extensive health care utilization. ${ }^{2}$ For that subset, there is a need to develop treatments that prevent symptoms and improve patient morbidity and long-term management.

Despite its singular name, the term, "asthma", actually encompasses a range of symptoms and diseases caused by distinct cellular mechanisms. ${ }^{4}$ While current guidelines classify this heterogeneous disease based on lung function, symptoms, and frequency of rescue bronchodilator use, ${ }^{5}$ efforts have been made to properly delineate asthma as distinct phenotypes. One such characterized phenotype is eosinophilic asthma, defined by the presence of eosinophils in the lungs. ${ }^{6}$ A subgroup of these patients maintains persistent eosinophilia in the airways and sputum even with conventional asthma therapy - termed steroid-resistant eosinophilic asthma. ${ }^{4,6}$ Many of these patients with eosinophilic asthma suffer significant morbidity and loss of quality 
of life despite using the currently available treatments. In this review, we discuss monoclonal antibodies targeting the biological activity of IL-5 in the treatment of difficult-tomanage patients with eosinophilic asthma.

\section{Eosinophils, IL-5, and asthma}

Eosinophils comprise $1 \%-6 \%$ of the white blood cells and are important defenders against parasitic infection. ${ }^{7}$ These cells are important mediators of the allergic inflammatory response, and they are significant players in the pathogenesis and severity of chronic inflammatory disorders of the airway including asthma. ${ }^{6,8}$ In fact, tissue eosinophilia is present in $40 \%-60 \%$ of patients with asthma, ${ }^{9}$ and blood and sputum eosinophilia parallel severity of disease for those with eosinophilic asthma. ${ }^{10,11}$ Eosinophils aid in the innate immune response triggered in the airway by environmental allergens, viral infections, and other extraneous stimuli, and activation of these cells can lead to tissue damage and remodeling. ${ }^{8,12}$ Through a battery of powerful proinflammatory mediators released from tissue eosinophils, including granule-derived basic proteins, lipid mediators, cytokines, and chemokines, these cells are responsible for inflammation of the airways, leading to hyperresponsiveness in addition to airway remodeling via fibrosis, angiogenesis, and thickening of airway walls (Figure 1). ${ }^{11,13}$ Conventional therapies with inhaled corticosteroids typically reduce total amounts of eosinophils in the airways of asthmatics. ${ }^{14}$ However, $\sim 50 \%$ of severe asthmatics, a group that constitutes $5 \%-10 \%$ of all asthma patients, have exacerbations and symptoms with persistent eosinophils in the airway despite taking high dose inhaled corticosteroids. ${ }^{15-17}$

IL-5 is the only known human eosinophilopoietin. ${ }^{7}$ As such, it plays an important role in allergic inflammation via the production, maturation, recruitment, differentiation, survival, and activation of eosinophils (Figure 1)..$^{818-21}$ In human ex vivo studies, exposure of peripheral blood eosinophils to IL-5 can prompt their activation leading to release of toxic granules. ${ }^{20}$ In mouse models, overexpression of IL-5 causes eosinophilic airway inflammation and airway hyperresponsiveness. ${ }^{21}$ Furthermore, blocking IL-5 in these mouse models leads to diminished eosinophilia and overall inflammation. ${ }^{22,23}$ In human disease, there is a correlation in the amount of IL-5 protein and mRNA expression isolated from bronchial mucosa and bronchoalveolar lavage fluid and evidence of asthma control. ${ }^{24-28}$ Taken together, these studies suggest that blocking IL-5 with a monoclonal antibody would diminish lung tissue eosinophilia and improve asthma severity. We have summarized the clinical trials for monoclonal IL-5 antibodies in Table 1.

\section{The history of mepolizumab: back to the drawing board}

Given the relationship of IL-5 to eosinophilia and asthma severity, targeting IL-5 became an obvious choice for treatment of severe asthma (Figure 1). Studies of IL-5 knockout

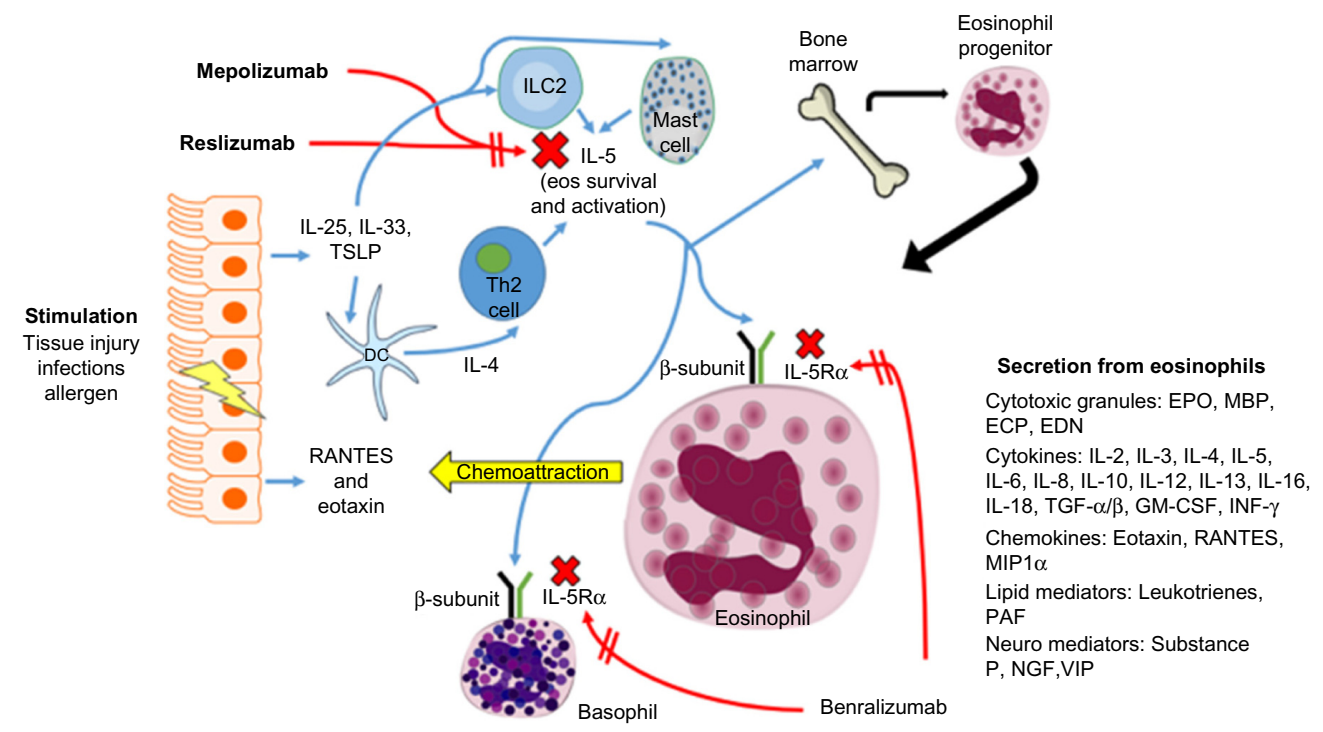

Figure I Eosinophil (eos) trafficking and maturation in asthma.

Notes: Stimulation at the epithelial cell surface leads to the generation of cytokines and chemokines that increase production of IL-5. Generation of IL-5 by Th2 cells, ILC2, and mast cells is essential in eosinophil survival and activation in peripheral tissue. This figure also shows the mechanisms of action for mepolizumab, reslizumab, and benralizumab, as well as the secretory products of eosinophils. Red arrows represent inhibitory pathways, while blue arrows are activating pathways.

Abbreviations: EDN, eosinophil-derived neurotoxin; ECP, eosinophil cationic protein; EPO, eosinophil peroxidase; IL, Interleukin; TGF- $\alpha / \beta$, transforming growth factor- $\alpha / \beta$; INF- $\gamma$, interferon gamma; RANTES, regulated upon activation, normal T cell expressed and secreted; MIPI $\alpha$, macrophage inflammatory proteins-I $\alpha$; PAF, platelet-activating factor; VIP, vasoactive intestinal peptide; NGF, nerve growth factor; TSLP, thymic stromal lymphopoietin; ILC2, innate lymphoid cells (type 2). 


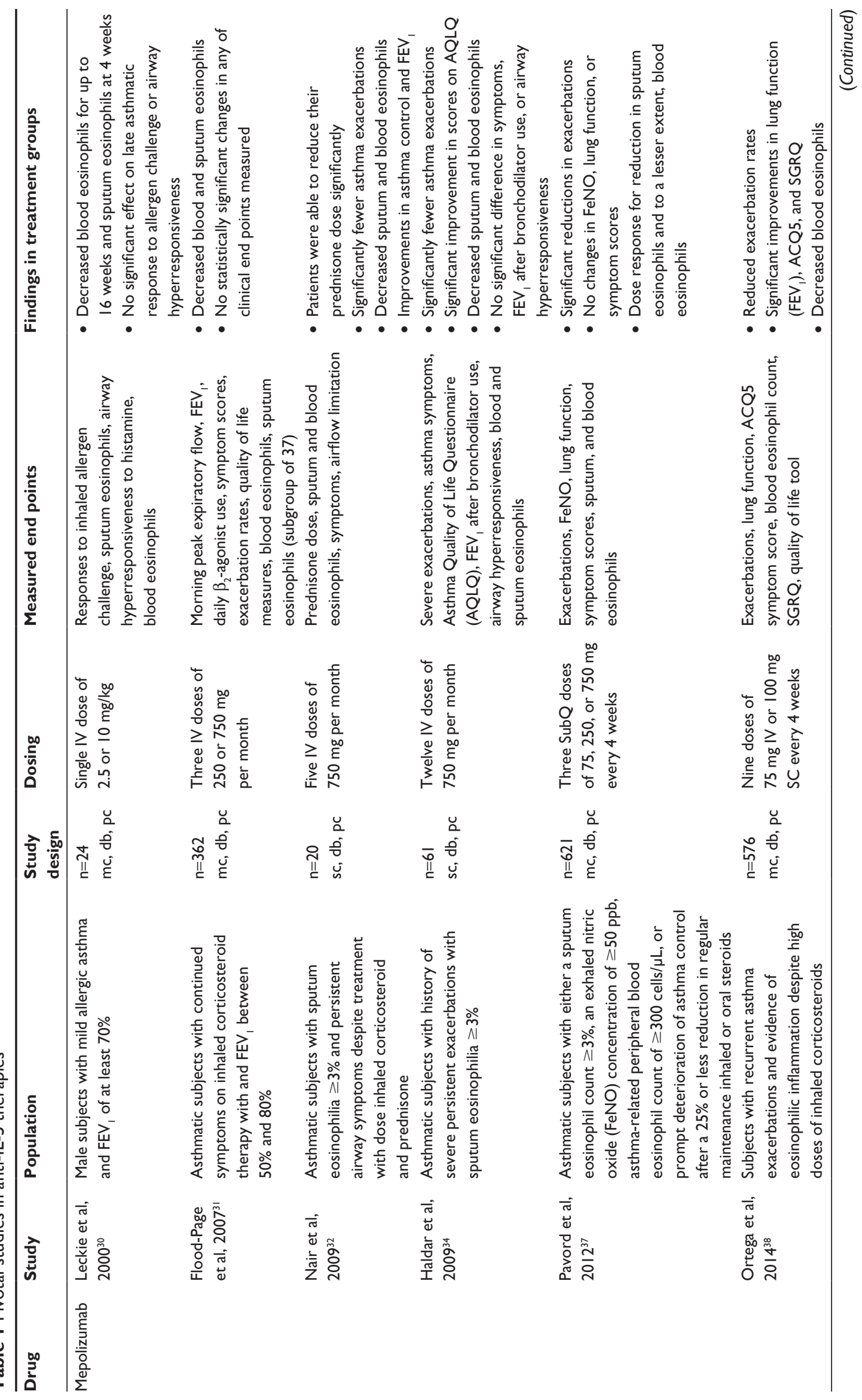




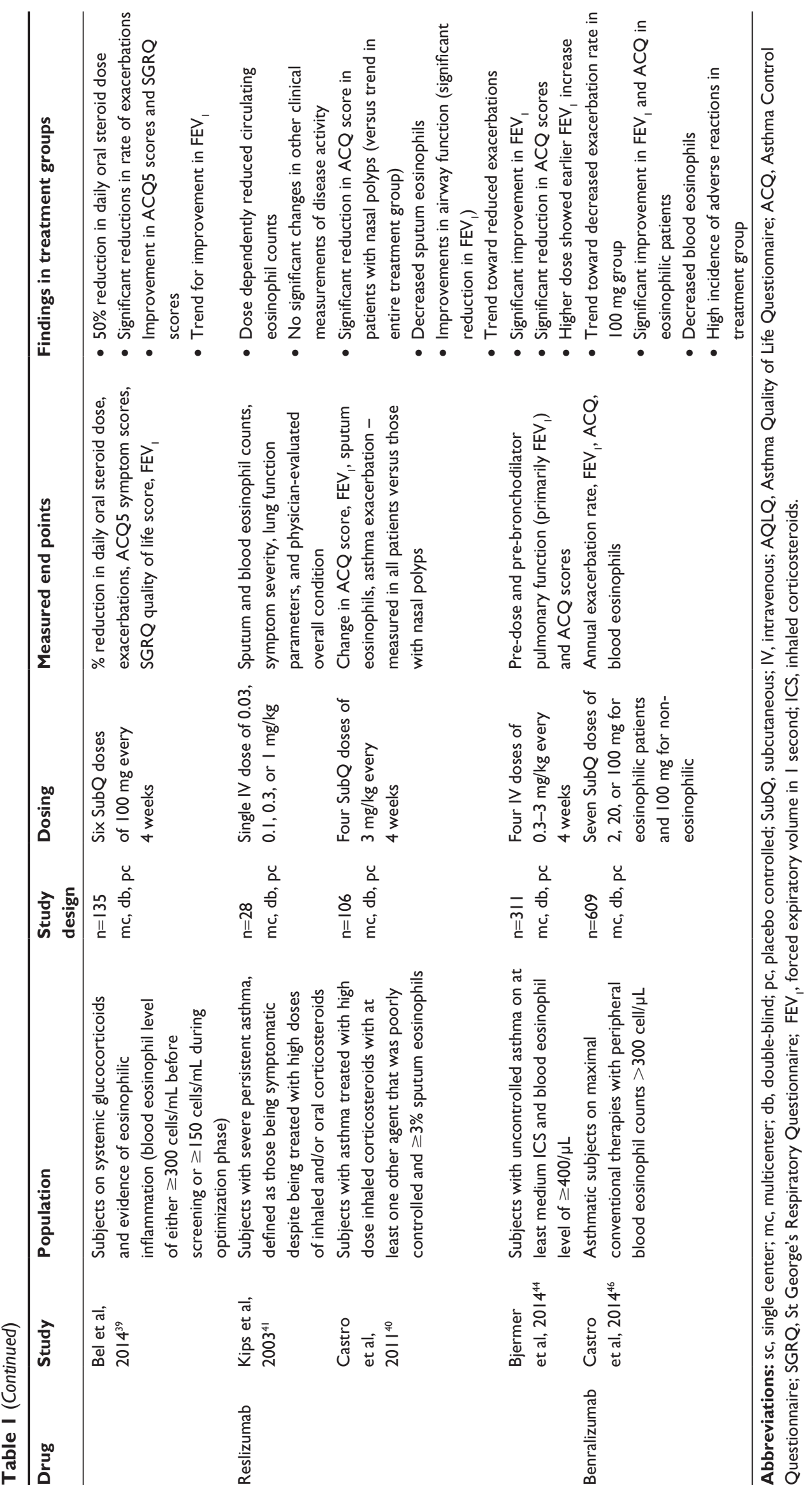


animals were encouraging. ${ }^{20,22}$ Preliminary studies with humans further reinforced the idea of targeting of IL-5 and therefore eosinophils. ${ }^{10,29}$ However, when studies began to shift to asthma symptoms and improvement of lung function, the IL-5 antibodies were disappointing as a solution for asthmatics, who continued to suffer from persistent symptoms despite maximized standard therapy. In order to learn from this history, we present data from these early experiments as follows.

Leckie et al studied mepolizumab, a monoclonal antibody against IL-5, and its effects on eosinophils, airway hyperresponsiveness, and the late phase allergic response, a process driven by eosinophils. This group found that mepolizumab effectively reduced the blood eosinophil count and this effect was maintained up to 16 weeks after the dose. Further, this drug reduced blood eosinophils after allergen challenge and decreased sputum eosinophils. The consequence of reduced eosinophilia in these subjects was predicted to be diminished airway hyperresponsiveness and late phase allergic responses. However, there was no significant effect observed on either outcome. The results of this study cast uncertainty on the exact relationship between the late phase allergic response, airway hyperresponsiveness, and eosinophilia. Upon closer examination of the population studied, the majority were men with history of intermittent wheezing and shortness of breath, diagnosed with mild allergic asthma with a forced expiratory volume in 1 second $\left(\mathrm{FEV}_{1}\right)$ of at least $70 \%$. There were no initial evaluations performed to determine the level of eosinophilia within these subjects prior to the onset of the study. ${ }^{30}$

In another study, Flood-Page et al studied two doses of mepolizumab versus placebo in asthmatic patients with continued symptoms on inhaled corticosteroid therapy with an $\mathrm{FEV}_{1}$ between $50 \%$ and $80 \%$. The measured end points for this study included blood and sputum eosinophils, airway hyperresponsiveness, and the late phase allergic response. As specific secondary end points, this group further evaluated change from baseline in domiciliary morning peak expiratory flow, changes from baseline $\mathrm{FEV}_{1}$, asthma summary symptom scores, use of rescue bronchodilator medications, quality of life scores, and asthma exacerbation rates. As with the study by Leckie et al, there was a significant, persistent decrease in blood and sputum eosinophils in the IL-5 monoclonal antibody groups compared to placebo. However, regardless of this observed reduction in eosinophilia, clinical effects were minimal. There were no statistically significant clinical benefits observed over the course of the study, including no apparent effect on airway hyperresponsiveness or late phase allergic responses after allergen challenge. Furthermore, there were no significant changes in $\mathrm{FEV}_{1}$ between treatment groups and all three groups had decreases in mean asthma summary symptom scores. Interestingly, the greatest decrease in mean asthma summary symptom scores was seen in the placebo group and there were no significant differences seen in rescue medication use or mean Asthma Quality of Life Questionnaire (AQLQ) scores. While not significant, a trend was observed of fewer asthma exacerbations in those subjects on the higher dose treatment with mepolizumab. ${ }^{31}$

Anti-IL-5 antibodies seemed destined to be placed on the back shelf as they did not seem to provide any clinical benefit in difficult-to-treat patients. However, these studies became the archetype of the importance of choosing the correct population and primary end points for study of particular drugs. With the advent of personalized medicine, some researchers saw an important phenotype that might respond much differently to these medications - the eosinophilic asthmatic. Equally as important for determining the utility of these medications in the appropriate population proved to be defining the correct outcome parameter(s) for the study. We will discuss these issues further in the following section.

\section{The history of mepolizumab: the importance of phenotype}

When anti-IL-5 therapies have been used in a patient population selected for eosinophils, results have been more promising. Nair et al evaluated mepolizumab in a group of patients selected for sputum eosinophilia (at least 3\%) and persistent airway symptoms despite treatment with high dose inhaled corticosteroid and prednisone. The primary end point for this study specifically examined the steroid-sparing effect of mepolizumab. The subjects who took mepolizumab were able to reduce oral steroids with an average reduction in prednisone of 11.9 to $3.9 \mathrm{mg}$ in the treatment group and a reduction from 10.7 to $6.4 \mathrm{mg}$ in the placebo arm. This reduction of prednisone dose was significantly different $(P=0.04)$. However, the final prednisone doses were not significantly different between groups. As in the previously mentioned studies, there was a significant reduction in eosinophils in circulation and sputum. However, unlike previous studies, in these subjects with high sputum eosinophils, improvements in asthma control were made as evidenced by improvement in scores on the Juniper Asthma Control Questionnaire (ACQ) and modest improvements in $\mathrm{FEV}_{1}$ that were maintained for 8 weeks. Furthermore, in those receiving mepolizumab, the median time to exacerbation was 20 weeks as compared to 12 weeks in the placebo group $(P=0.003)$. In ten placebo subjects, there were 12 exacerbations, and, at the time of 
exacerbation, nine had sputum eosinophilia. In the mepolizumab group, only one subject had an asthma exacerbation. There was also improvement in symptoms; however, this was not statistically significant. These findings were counter to the previous studies that had negative results in subjects not selected for eosinophilia, drawing attention to the need to identify biomarkers that would predict response to therapy for these medications. ${ }^{32}$

Further support of this idea was shown by Haldar et al. ${ }^{33}$ This study was performed with subjects who had a history of severe persistent exacerbations with eosinophilic asthma as evidenced by sputum eosinophilia $\geq 3 \%$. In this study, the number of severe exacerbations during treatment was the primary outcome measure with other measured outcomes, including change in asthma symptoms, AQLQ, FEV 1 after use of bronchodilator, airway hyperresponsiveness, and number of eosinophils in the blood and sputum. As with all previous studies of anti-IL-5, blood and sputum eosinophils were significantly decreased. Over the 50-week period, patients receiving mepolizumab had significantly fewer asthma exacerbations than those in the placebo group. Per subject, there were on average 2.0 severe exacerbations in the mepolizumab group compared to 3.4 in placebo. Closer examination of hospital admissions during exacerbations revealed only three in the mepolizumab group, with eleven in the placebo group. This decrease in number of admissions was not statistically significant, though arguably was clinically significant; however, the length of hospital stay during exacerbations was significantly less for the mepolizumab group contrasted with placebo. Further, those in the mepolizumab group had significant improvement in scores on the AQLQ. The investigators also evaluated computed tomography images of the lungs to scrutinize airway wall thickness and total wall area, and these were both decreased in those receiving mepolizumab. Others have shown that mepolizumab decreases extracellular matrix protein deposition, which might explain improvements in airway wall thickness. ${ }^{33}$ Nonetheless, there was no significant difference in symptoms, $\mathrm{FEV}_{1}$ after bronchodilator use, airway hyperresponsiveness, and rescue bronchodilator use. ${ }^{34}$

The DREAM study by Pavord et al, ${ }^{35}$ was a multicenter, double-blind placebo-controlled trial of subjects with severe asthma. Subjects were enrolled if they had one of the following: either a sputum eosinophil count $\geq 3 \%$, an exhaled nitric oxide (FeNO) concentration of $\geq 50 \mathrm{ppb}$, asthmarelated peripheral blood eosinophil count of $\geq 300$ cells $/ \mu \mathrm{L}$, or prompt deterioration of asthma control after a $25 \%$ or less reduction in regular maintenance inhaled or oral steroids.
Subjects were provided with one of three different doses of mepolizumab or placebo. Ultimately, 616 participants completed the treatment with 13 infusions at 4-week intervals. The primary outcome of the study was the rate of clinically significant asthma exacerbations occurring in the 52 weeks of treatment and 4 weeks after completion of treatment. Clinically significant asthma exacerbations were defined as "worsening of asthma requiring the use of oral corticosteroids for 3 or more days, admission, or a visit to the emergency department". This study again showed diminished blood and sputum eosinophils during the course of treatment for the active group. In this study, $75 \mathrm{mg}$ of intravenous (IV) mepolizumab every month, the lowest dose in the study, decreased clinically significant asthma exacerbations by $48 \%$ during the course of treatment. The $250 \mathrm{mg}$ and the $750 \mathrm{mg}$ also improved asthma exacerbations, but with no more significant gains than that obtained by $75 \mathrm{mg}$. There are two possibilities as to why this might be the case. First, it is possible that a certain proportion of exacerbations are not eosinophil dependent, despite the fact that they are occurring in patients with high numbers of eosinophils. Alternatively, this finding may represent that mepolizumab does not completely abolish airway eosinophils. We speculate that this may be the result of IL-5-independent prolongation of survival and activation of eosinophils in the tissues by GM-CSF or IL-3. ${ }^{36,37}$ There were only small effects on $\mathrm{FEV}_{1}$ and ACQ scores, which were not statistically significant. The authors postulate that day-to-day symptoms are separate from exacerbations associated with severe asthma, and they may "require different management strategies".

In 2014, Ortega et al studied 576 patients with recurrent asthma exacerbations and evidence of eosinophilic inflammation despite high doses of inhaled corticosteroids. Subjects were randomized into one of three groups: $75 \mathrm{mg}$ IV mepolizumab, $100 \mathrm{mg}$ subcutaneous mepolizumab, or placebo. They found that both IV and subcutaneous mepolizumab reduced exacerbation rates ( $47 \%$ and $53 \%$, respectively) compared to those receiving placebo. Severe exacerbations requiring an emergency department visit were decreased by $32 \%$ in the IV group and $61 \%$ in the subcutaneous group. Both $\mathrm{FEV}_{1}$ and AQLQ scores increased for both the IV and subcutaneous mepolizumab as compared to placebo. ${ }^{38}$

Finally, Bel et al studied mepolizumab with the primary outcome of its ability to spare oral corticosteroids. In this study, 135 subjects were randomized to receive either $100 \mathrm{mg}$ mepolizumab subcutaneously or placebo. The mepolizumab group was able to reduce corticosteroid doses by $50 \%$ compared to no reduction in the placebo group. The treatment 
group also showed a relative reduction in the rate of asthma exacerbations, underscoring the importance of eosinophils in exacerbations of asthma. ${ }^{39}$

\section{The history of mepolizumab: the importance of choosing outcomes}

In the studies mentioned earlier, the authors consistently found that mepolizumab was effective at improving rates of exacerbations in the eosinophilic asthmatic. In contrast, there were few to no consistent effects on quality of life, nor were there consistent changes in objective measures of asthma, including $\mathrm{FEV}_{1}$, airway hyperreactivity, symptoms, or rescue inhaler use. ${ }^{32,34,37-39}$ These findings suggest that the cellular inflammatory mechanisms driving day-to-day symptoms are separate from those driving exacerbations, with exacerbations more consistently reflecting an eosinophildriven process. Furthermore, these observations should also drive the expectations that clinicians will provide to their patients when offering these types of medications when they are approved and use them primarily targeted to patients with a history of frequent exacerbations. Finally, these studies drive the overarching point that along with choosing the right asthma phenotype, choosing the right primary outcome for clinical studies is paramount for determining the efficacy of a given intervention.

\section{Reslizumab and asthma}

Reslizumab, an IgG4/ $\kappa$ humanized monoclonal antibody, was also created to neutralize circulating IL-5 and prevent it from binding eosinophils (Figure 1). This particular monoclonal antibody has been studied in both United States and Europe for the treatment of moderate to severe asthma with persistent eosinophilia despite typical treatments. It is given IV every 4 weeks. ${ }^{40}$

As with mepolizumab, early clinical trials of reslizumab in the treatment of severe asthma patients with uncontrolled symptoms were not promising. ${ }^{41}$ In fact, these trials also showed no improvement in $\mathrm{FEV}_{1}$ or symptoms. However, researchers did again report a significant and profound reduction in circulating eosinophils. Phase II trials emphasized evidence of eosinophils (sputum eosinophils $>3 \%$ ) for enrollment, and, as with mepolizumab, these studies were more successful with regards to treatment end points. The reslizumab cohort exhibited a decrease in sputum eosinophilia as well as a modest but nonsignificant improvement in asthma control by ACQ. However, this trial also recognized an important subgroup of asthmatics with nasal polyposis, which showed robust improvement of asthma symptoms $(P=0.012) .{ }^{42}$ The association with chronic rhinosinusitis with nasal polyposis is almost certain to define a phenotype of asthmatics with disease driven by eosinophilic inflammation. However, efficacy in this cohort may also reflect the ability of IL-5 antagonism to improve chronic rhinosinusitis with nasal polyposis ${ }^{43}$ with secondary improvements in asthma in response to reduced sinonasal inflammation.

Phase III trials in Europe compared the efficacy of reslizumab to placebo as an add-on therapy to recommended asthma medications in subjects with moderate-to-severe persistent disease and elevated blood eosinophils $(>400 / \mu \mathrm{L})$. This study showed significantly improved $\mathrm{FEV}_{1}$ and ACQ score (treatment difference vs placebo: 115 and $160 \mathrm{~mL}$, $\mathrm{FEV}_{1}$ and 0.238 and $0.359 \mathrm{ACQ}$, respectively). ${ }^{44}$ In another study performed in the United States, Castro et al evaluated reslizumab in subjects with elevated sputum eosinophils $(>3 \%)$. The primary end point of this study was enhanced quality of life as suggested by improved ACQ scores. This group showed significant improved ACQ and FEV 1 in the treatment group. In a subgroup post hoc analysis, this group evaluated those subjects with nasal polyps and showed even more improvement in ACQ in this group compared to those without nasal polyps. ${ }^{40}$ In those with nasal polyposis, it was previously suggested the best predictor for nasal symptom response to reslizumab was nasal IL-5 levels. ${ }^{43}$

\section{Benralizumab and asthma}

Benralizumab takes a slightly different approach to IL-5 antagonism by blocking IL-5R $\alpha$. As with the other monoclonal antibodies to IL-5, this drug significantly depletes blood and airway eosinophils. Basophils also have IL-5R on their surface and are thereby also targeted by benralizumab. As a unique mechanism of action compared to mepolizumab or reslizumab, benralizumab facilitates antibody-dependent cell-mediated cytotoxicity of both basophils and eosinophils, allowing for an active immune response to these cells rather than the passive approach of the other two medications, which primarily act to block their differentiation and survival. ${ }^{45}$ In Phase IIB trials of subjects with severe asthma on maximal conventional therapies with peripheral blood eosinophil counts $>300$ cell $/ \mu \mathrm{L}$, benralizumab reduced exacerbation rates compared with placebo at both 20 and $100 \mathrm{mg}$ doses. ${ }^{46}$ These findings again emphasize the importance of choosing the correct primary end point, one driven by eosinophilic inflammation.

\section{Personalized medicine}

Medication costs for asthma, especially for monoclonal antibodies, have skyrocketed. Phase I studies of reslizumab and 
mepolizumab have made it abundantly clear that biomarkers are necessary to predict which subset of severe asthmatics will respond best to these medications. The presence of sputum and blood eosinophils have been used to identify subjects who go on to demonstrate improvements in frequency of exacerbations and to a lesser extent in quality of life and other parameters. Unfortunately, induced sputum is impractical and is almost certain not to achieve US Food and Drug Administration (FDA) endorsement as a basis for recommending use of these agents and circulating eosinophilia may have only modest value in predicting airway pathology. In this section, we will discuss alternative biomarkers that could be used to predict responses to anti-IL-5 therapies.

With the advent of easier to use and more cost-effective ways to measure expired FeNO, many practitioners are turning to this test to aid in the treatment of patients with asthma. Levels of FeNO correlate well with numbers of eosinophils in the lungs in a number of studies. ${ }^{47}$ In a study by Schleich et al, a FeNO $>21$ correlated well with having $\geq 3 \%$ sputum eosinophils and as such could theoretically be used as a less invasive biomarker supporting use of IL-5 antagonizing agents. ${ }^{48}$ These findings have been confirmed in a study by Westerhof et al. ${ }^{49}$ However, in a study by Wagener et al, FeNO corresponded to sputum eosinophilia in only $78 \%$ of subjects, ${ }^{50}$ a result also observed in the DREAM study mentioned earlier. ${ }^{37}$

Serum periostin, a marker that reflects the biological activity of IL-13, has been used to predict responders to lebrikizumab (anti-IL-13 monoclonal antibody). However, there is conflicting evidence regarding its ability to distinguish eosinophilic asthma. In a study by Jia et al, serum periostin levels were the single best predictor of airway eosinophilia, even when compared to FeNO. ${ }^{51}$ On the other hand, both Westerhof et al and Wagener et al show that neither periostin nor total IgE were able to distinguish eosinophilic from noneosinophilic airway inflammation. ${ }^{49,50}$ Given the conflicting information, it seems that further evidence is necessary to determine whether periostin discriminates between eosinophilic and non-eosinophilic asthma.

Nasal IL-5 levels have also been proposed to classify asthmatic subjects with regard to their likelihood of responding to anti-IL-5 therapies. As mentioned, in their study of reslizumab, Castro et al showed that the subgroup of patients with nasal polyps showed even more improvement in ACQ. ${ }^{40,43}$ Alternatively, other nasal wash markers that indirectly reflect eosinophilia might define responders to anti-IL-5 therapies, such as eosinophil-recruiting chemokines (CCL11 [eotaxin] or CCL5 [RANTES]) or eosinophil-secreted products such as EDN. However, none of these biomarkers is fully accepted as a direct marker of lung eosinophilia. The use of these approaches is further constrained by the absence of validated methodologies for either collecting samples or quantifying results.

\section{Conclusion}

Asthma is a heterogeneous disease, and, through time and clinical experience, it has become clear that one "size" does not fit all when it comes to therapies. Guideline-based therapies work for the majority of patients; however, there are some who continue to have symptoms despite typical recommended treatments. One specific asthma phenotype is defined by the presence of persistent eosinophilic inflammation despite high dose inhaled corticosteroids therapy, and many of these patients have multiple exacerbations each year with diminished quality of life. With the advancement of monoclonal antibodies to IL-5 and its receptor and the development of specific biomarkers that define eosinophilia, reslizumab, mepolizumab, and benralizumab are becoming viable options to aid in treatment of these patients. In fact, an FDA advisory committee has recently approved the use of mepolizumab for patients with eosinophilic severe asthma. While expensive, these antibodies have shown great promise in patients with persistent eosinophilia despite high dose inhaled corticosteroids therapy, particularly in reducing the number of exacerbations. Moving forward, it will be important to develop more specific and easier to obtain biomarkers, which will provide the best information with regard to who will respond to these therapies. Further, with the generation of several new monoclonal antibodies for use with asthma, studies need to be performed that distinguish which patients will respond to particular antibodies, both within and between classes (ie, Who will respond to mepolizumab vs benralizumab? Who will respond to mepolizumab vs lebrikizumab?) These anti-IL-5 monoclonal antibodies will very soon provide another treatment in the asthma toolbox.

\section{Disclosure}

Dr Megan F Patterson and Dr Larry Borish do not report any conflicts of interest in this work. Dr Joshua L Kennedy has grants to study asthma from the National Institutes of Health (KL2TR000063, UL1TR000039, P20GM103625) and the Arkansas Biosciences Institute.

\section{References}

1. Akinbami LJ, Moorman JE, Bailey C, et al. Trends in asthma prevalence, health care use, and mortality in the United States, 2001-2010. NCHS Data Brief. 2012;(94):1-8. 
2. Akinbami LJ, Sullivan SD, Campbell JD, et al. Asthma outcomes: healthcare utilization and costs. J Allergy Clin Immunol. 2012;129 (3 Supp1):S49-S64.

3. Blackwell DL LJ, Clarke TC. Summary health statistics for US adults: National Health Interview Survey, 2012. National Center for Health Statistics. Vital Health Stat. 2014;(260):1-161.

4. Moore WC, Meyers DA, Wenzel SE, et al. Identification of asthma phenotypes using cluster analysis in the Severe Asthma Research Program. Am J Respir Crit Care Med. 2010;181(4):315-323.

5. National Asthma Education and Prevention Program. Expert panel report 3 (EPR-3): guidelines for the diagnosis and management of asthma-summary report 2007. J Allergy Clin Immunol. 2007;120 (5 Suppl):S94-S138.

6. Nair P. What is an "eosinophilic phenotype" of asthma? J Allergy Clin Immunol. 2013;132(1):81-83.

7. Stone KD, Prussin C, Metcalfe DD. IgE, mast cells, basophils, and eosinophils. J Allergy Clin Immunol. 2010;125(2 Supp1 2):S73-S80.

8. Rothenberg ME, Hogan SP. The eosinophil. Annu Rev Immunol. 2006;24:147-174.

9. Zhang JY, Wenzel SE. Tissue and BAL based biomarkers in asthma. Immunol Allergy Clin North Am. 2007;27(4):623-632; vi.

10. Bousquet J, Chanez P, Lacoste JY, et al. Eosinophilic inflammation in asthma. N Engl J Med. 1990;323(15):1033-1039.

11. Wardlaw AJ, Brightling CE, Green R, Woltmann G, Bradding P, Pavord ID. New insights into the relationship between airway inflammation and asthma. Clin Sci (Lond). 2002;103(2):201-211.

12. Lee JJ, Jacobsen EA, McGarry MP, Schleimer RP, Lee NA. Eosinophils in health and disease: the LIAR hypothesis. Clin Exp Allergy. 2010; 40(4):563-575.

13. Nissim Ben Efraim AH, Levi-Schaffer F. Tissue remodeling and angiogenesis in asthma: the role of the eosinophil. Ther Adv Respir Dis. 2008;2(3):163-171.

14. Wenzel SE, Schwartz LB, Langmack EL, et al. Evidence that severe asthma can be divided pathologically into two inflammatory subtypes with distinct physiologic and clinical characteristics. Am J Respir Crit Care Med. 1999;160(3):1001-1008.

15. Woodruff PG, Modrek B, Choy DF, et al. T-helper type 2-driven inflammation defines major subphenotypes of asthma. Am J Respir Crit Care Med. 2009;180(5):388-395.

16. Chung KF, Wenzel SE, Brozek JL, et al. International ERS/ATS guidelines on definition, evaluation and treatment of severe asthma. Eur Respir J. 2014;43(2):343-373.

17. Wenzel S. Severe asthma in adults. Am J Respir Crit Care Med. 2005; 172(2):149-160.

18. Sehmi R, Wardlaw AJ, Cromwell O, Kurihara K, Waltmann P, Kay AB. Interleukin-5 selectively enhances the chemotactic response of eosinophils obtained from normal but not eosinophilic subjects. Blood. 1992;79(11):2952-2959.

19. Garcia G, Taille C, Laveneziana P, Bourdin A, Chanez P, Humbert M Anti-interleukin-5 therapy in severe asthma. Eur Respir Rev. 2013;22(129):251-257.

20. Gleich GJ. Mechanisms of eosinophil-associated inflammation. J Allergy Clin Immunol. 2000;105(4):651-663.

21. Yamaguchi Y, Suda T, Suda J, et al. Purified interleukin 5 supports the terminal differentiation and proliferation of murine eosinophilic precursors. J Exp Med. 1988;167(1):43-56.

22. Foster PS, Hogan SP, Ramsay AJ, Matthaei KI, Young IG. Interleukin 5 deficiency abolishes eosinophilia, airways hyperreactivity, and lung damage in a mouse asthma model. $J$ Exp Med. 1996;183(1): 195-201.

23. Lee JJ, McGarry MP, Farmer SC, et al. Interleukin-5 expression in the lung epithelium of transgenic mice leads to pulmonary changes pathognomonic of asthma. J Exp Med. 1997;185(12): 2143-2156.

24. Hamid Q, Azzawi M, Ying S, et al. Expression of mRNA for interleukin-5 in mucosal bronchial biopsies from asthma. J Clin Invest. 1991;87(5):1541-1546.
25. Humbert M, Corrigan CJ, Kimmitt P, Till SJ, Kay AB, Durham SR. Relationship between IL-4 and IL-5 mRNA expression and disease severity in atopic asthma. Am J Respir Crit Care Med. 1997;156 (3 Pt 1):704-708.

26. Lamblin C, Gosset P, Salez F, et al. Eosinophilic airway inflammation in nasal polyposis. J Allergy Clin Immunol. 1999;104(1):85-92.

27. Robinson D, Hamid Q, Bentley A, Ying S, Kay AB, Durham SR. Activation of CD4+ T cells, increased TH2-type cytokine mRNA expression, and eosinophil recruitment in bronchoalveolar lavage after allergen inhalation challenge in patients with atopic asthma. J Allergy Clin Immunol. 1993;92(2):313-324.

28. Robinson DS, Ying S, Bentley AM, et al. Relationships among numbers of bronchoalveolar lavage cells expressing messenger ribonucleic acid for cytokines, asthma symptoms, and airway methacholine responsiveness in atopic asthma. J Allergy Clin Immunol. 1993;92(3):397-403.

29. Shi HZ, Xiao CQ, Zhong D, et al. Effect of inhaled interleukin-5 on airway hyperreactivity and eosinophilia in asthmatics. Am J Respir Crit Care Med. 1998;157(1):204-209.

30. Leckie MJ, ten Brinke A, Khan J, et al. Effects of an interleukin-5 blocking monoclonal antibody on eosinophils, airway hyper-responsiveness, and the late asthmatic response. Lancet. 2000;356(9248):2144-2148.

31. Flood-Page P, Swenson C, Faiferman I, et al. A study to evaluate safety and efficacy of mepolizumab in patients with moderate persistent asthma. Am J Respir Crit Care Med. 2007;176(11):1062-1071.

32. Nair P, Pizzichini MM, Kjarsgaard M, et al. Mepolizumab for prednisone-dependent asthma with sputum eosinophilia. NEngl J Med. 2009;360(10):985-993.

33. Flood-Page P, Menzies-Gow A, Phipps S, et al. Anti-IL-5 treatment reduces deposition of ECM proteins in the bronchial subepithelial basement membrane of mild atopic asthmatics. J Clin Invest. 2003; 112(7):1029-1036.

34. Haldar P, Brightling CE, Hargadon B, et al. Mepolizumab and exacerbations of refractory eosinophilic asthma. $N$ Engl $J$ Med. 2009;360(10):973-984.

35. Esnault S, Kelly EA, Shen ZJ, Johansson MW, Malter JS, Jarjour NN. IL-3 maintains activation of the p90S6K/RPS6 pathway and increases translation in human eosinophils. J Immunol. 2015; 195(6):2529-2539.

36. Esnault S, Malter JS. Granulocyte macrophage-colony-stimulating factor mRNA is stabilized in airway eosinophils and peripheral blood eosinophils activated by TNF-alpha plus fibronectin. J Immunol. 2001;166(7):4658-4663.

37. Pavord ID, Korn S, Howarth P, et al. Mepolizumab for severe eosinophilic asthma (DREAM): a multicentre, double-blind, placebocontrolled trial. Lancet. 2012;380(9842):651-659.

38. Ortega HG, Liu MC, Pavord ID, et al. Mepolizumab treatment in patients with severe eosinophilic asthma. $N$ Engl J Med. 2014; 371(13):1198-1207.

39. Bel EH, Wenzel SE, Thompson PJ, et al. Oral glucocorticoid-sparing effect of mepolizumab in eosinophilic asthma. N Engl J Med. 2014; 371(13):1189-1197.

40. Castro M, Mathur S, Hargreave F, et al. Reslizumab for poorly controlled, eosinophilic asthma: a randomized, placebo-controlled study. Am J Respir Crit Care Med. 2011;184(10):1125-1132.

41. Kips JC, O'Connor BJ, Langley SJ, et al. Effect of SCH55700, a humanized anti-human interleukin-5 antibody, in severe persistent asthma: a pilot study. Am J Respir Crit Care Med. 2003;167(12):1655-1659.

42. Bateman ND, Shahi A, Feeley KM, Woolford TJ. Activated eosinophils in nasal polyps: a comparison of asthmatic and non-asthmatic patients. Clin Otolaryngol. 2005;30(3):221-225.

43. Gevaert P, Lang-Loidolt D, Lackner A, et al. Nasal IL-5 levels determine the response to anti-IL-5 treatment in patients with nasal polyps. J Allergy Clin Immunol. 2006;118(5):1133-1141.

44. Bjermer CL, Jorge Maspero, Monika Ciesielska, Christopher O’Brien, James Zangrilli. A randomized phase 3 study of the efficacy and safety of reslizumab in subjects with asthma with elevated eosinophils. European Respiratory Journal. 2014;44(58):299. 
45. Ghazi A, Trikha A, Calhoun WJ. Benralizumab - a humanized mAb to IL-5Ralpha with enhanced antibody-dependent cell-mediated cytotoxicity - a novel approach for the treatment of asthma. Expert Opin Biol Ther. 2012;12(1):113-118.

46. Castro M, Wenzel SE, Bleecker ER, et al. Benralizumab, an antiinterleukin 5 receptor alpha monoclonal antibody, versus placebo for uncontrolled eosinophilic asthma: a phase $2 \mathrm{~b}$ randomised dose-ranging study. Lancet Respir Med. 2014;2(11):879-890.

47. Jatakanon A, Lim S, Kharitonov SA, Chung KF, Barnes PJ. Correlation between exhaled nitric oxide, sputum eosinophils, and methacholine responsiveness in patients with mild asthma. Thorax. 1998;53(2):91-95.
48. Schleich FN, Seidel L, Sele J, et al. Exhaled nitric oxide thresholds associated with a sputum eosinophil count $>/=3 \%$ in a cohort of unselected patients with asthma. Thorax. 2010;65(12):1039-1044.

49. Westerhof GA, Korevaar DA, Amelink M, et al. Biomarkers to identify sputum eosinophilia in different adult asthma phenotypes. Eur Respir J. 2015;46(3):688-696.

50. Wagener AH, de Nijs SB, Lutter R, et al. External validation of blood eosinophils, $\mathrm{FE}(\mathrm{NO})$ and serum periostin as surrogates for sputum eosinophils in asthma. Thorax. 2015;70(2):115-120.

51. Jia G, Erickson RW, Choy DF, et al. Periostin is a systemic biomarker of eosinophilic airway inflammation in asthmatic patients. J Allergy Clin Immunol. 2012;130(3):647-654. e10.

\section{Publish your work in this journal}

The Journal of Asthma and Allergy is an international, peer-reviewed open-access journal publishing original research, reports, editorials and commentaries on the following topics: Asthma; Pulmonary physiology; Asthma related clinical health; Clinical immunology and the immunological basis of disease; Pharmacological interventions and

\section{Dovepress}

new therapies. Issues of patient safety and quality of care will also be considered. The manuscript management system is completely online and includes a very quick and fair peer-review system, which is al easy to use. Visit http://www.dovepress.com/testimonials.php to read real quotes from published authors.

Submit your manuscript here: http://www.dovepress.com/journal-of-asthma-and-allergy-journal 